\title{
A Frequency Control Method for Islanded Microgrid Based on Feedforward and Feedback
}

\author{
Qiang Zhang ${ }^{1, ~ a}$, Kaifeng Zhang ${ }^{1, b}$, Chunyu Chen ${ }^{1, c}$, Kun Yuan ${ }^{1, \mathrm{~d}}$ and Xiang \\ Xiao ${ }^{1, e}$
}

${ }^{1}$ Key Laboratory of Measurement and Control of CSE School of Automation, Southeast University, Nanjing, China

a18751805115@163.com, bkaifengzhang@seu.edu.cn, ${ }^{c} 1551437792 @ q q . c o m$, d101010918@seu.edu.cn, ${ }^{e} 825692174 @ q q . c o m$

Keywords: Islanded microgrid; Frequency control; Feedforward and feedback control; New energy; Resources allocation.

Abstract. In this paper, a new frequency control strategy is proposed for islanded microgrid. Due to characteristics of islanded microgrid, the load variation and the new energy disturbance can be predicted by the ultra-short-term prediction. By using feedforward control method, the load disturbance can be roughly adjusted in advance. However, load variation and new energy disturbance in islanded microgrid cannot be precisely predicted and there must be prediction error. Therefore, feedback control is used to eliminate prediction error. Specifically, considering characteristics of different units, wind turbines and diesel engines are used in feedforward control part, and energy storage systems in the feedback control part. Besides, this paper proposes an allocation method on feedforward and feedback resources, which can improve resources utilization to a certain extent. Simulation results show that the proposed control method can improve the frequency control effect for islanded microgrid.

\section{Introduction}

The decentralization and diversification of electricity load increase the maintenance cost and control difficulty of grid construction. Microgrid technology becomes an effective means to solve the problem of decentralized power supply demand [1]. Microgrid is a system composed of load and micro-power, which can provide both power and heat. It is a single controlled unit with respect to the external large-scale power grid, and can meet the requirements of power quality and power supply security [2].

Microgrid can integrate a variety of distributed power supply for local load. It can not only solve the problem of power supply in remote areas, but also give full play to the advantages of renewable energy to promote renewable energy development. The diversity of distributed power supply and the fluctuation of renewable energy in the microgrid have a great impact on the safety and stability of the microgrid system, and limit the rapid development of the microgrid [1].

At present, on islanded microgrid frequency control, domestic and foreign researches can be divided into two aspects. One of them is about the control strategy based on droop control $[3,4,5]$, another is about the micro-power coordination control strategy [6,7,8,9]. Droop control is the primary means of islanded microgrid frequency control, but the ability of droop control to suppress disturbance is very weak. When the disturbance of system is so frequent, the islanded microgrid frequency will fluctuate constantly, which will make system very unstable. Due to the volatility of 
new energy power supply and the relatively slow response of fuel units, islanded microgrid largely relies on energy storage systems, which respond more quickly, but cost much. However, the coordination control strategy among micro-power supplies has not effectively reduced the pressure of energy storage systems [10].

Based on the analysis of merits and demerits of islanded microgrid frequency control methods above, this paper proposes the feedforward and feedback frequency control method.

In fact, the feedforward and feedback control method has been widely used in many control systems, such as the hydraulic power system [11], vehicle stability control system [12], numerical control machine tool system [13], current harmonic compensation [14], fans pitch angle control [15] and so on. In large power grids, the grid dispatch part can be similar to the feedforward part, while the grid control part is still using the traditional load frequency control, and does not involve feedforward control. Further, for the islanded microgrid frequency control, the feedforward and feedback control strategy has not been studied and used. Due to characteristics of islanded microgrid itself, load and new energy disturbance can be predicted in the ultra-short-term prediction, so that load disturbance can be roughly adjusted by the feed-forward part in advance, which can reduce pressure of energy storage systems. The load and the new energy disturbance are random, and there must be prediction error. Hence, the feedback part with energy storage systems needs to be added to compensate the prediction error. By using the feedforward and feedback method to control the islanded microgrid frequency, on the one hand, the burden of feedback control is greatly reduced, on the other hand, the accuracy requirement of the feedforward control model is reduced [16], and eliminate the error at the same time. As a result, the islanded microgrid frequency is effectively controlled.

\section{Overall Research Idea}

In islanded microgrid, the fluctuation of wind power and load disturbance is large, which makes it difficult to control the frequency. At the same time, new energy is limited in islanded microgrid. In order to make full use of local resources and obtain greater economic benefits, resource allocation must be optimized to save power generation costs.

The microgrid frequency fluctuation reflects the active balance of the microgrid system, and the active balance of the system is determined by the size of the active output of generators and load demand. When there is active unbalance in the microgrid system, each generator has to regulate its output to meet the load demand.

In this paper, a frequency control method based on feedforward and feedback is proposed for the islanded microgrid. The overall idea is shown in Fig 1. The whole is divided into two parts: feedforward and feedback. Wind turbines and diesel generators are used in the feedforward part, mainly in order to roughly adjust load disturbance by predicting the load and the wind power, while energy storage systems are used in feedback part, mainly in order to quickly compensate for the load and wind power prediction error. Finally, this paper proposes a method about feedforward and feedback resources allocation by analyzing historical data to improve resources utilization. 


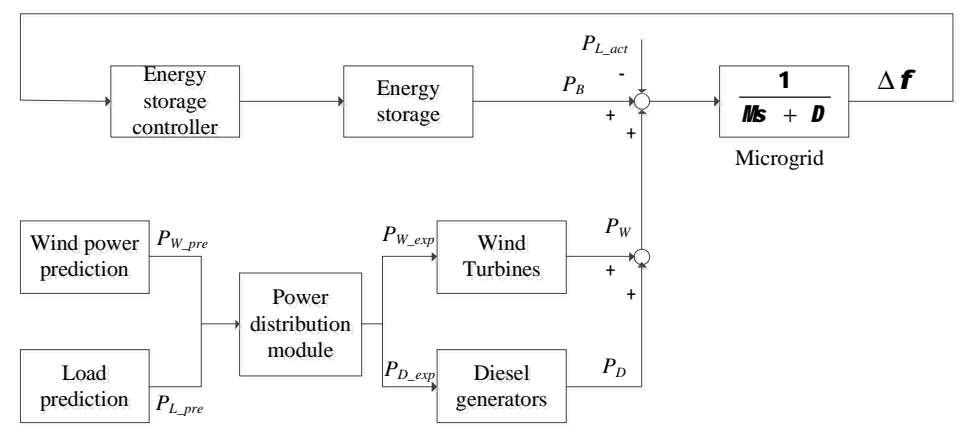

Fig. 1 Overall idea in this paper

\section{Ultra-short-term Prediction Method of Wind Power and Load.}

With the development of the prediction technology, wind power prediction and load prediction are playing an important role in grids control. Among the prediction methods, the time series prediction method is based on the trend extrapolation of the target variable itself over time. This method has the advantages of simple model and small calculation, because there is no necessary to consider more meteorological information. But, when modeling, it needs a lot of historical data, and its robustness is poor. Also, the prediction accuracy decreases rapidly with the increase of the prediction time. Hence, it is suitable for the ultra-short-term prediction of wind farms with limited meteorological information. Classical time series prediction method includes continuous method, moving average method and auto regressive moving average method [17].

Among them, the moving average method means that, as the time series moves at a constant width window, the dynamic mean value of each item is taken as the prediction value of the next time. It is suitable for the system, which has small fluctuation. The calculation formula is as Eq. 1.

$$
X(t)=(X(t-1)+X(t-2)+X(t-3)+\ldots+X(t-N)) / N
$$

Where $X(t)$ is prediction value of time $t, X(t-1)$ to $X(t-n)$ are the actual values of $\mathrm{n}$ historical moments before time $t$.

In this paper, the moving average method is used to predict the wind power and the load.

\section{Design of Control Method}

Design of Feedforward Part. The feedforward part makes full use of prediction technique to roughly adjust the load disturbance, which can greatly reduce the burden of feedback part. In this part, wind turbines and diesel generators are used as the main energy source to control load disturbance in advance. For the wind power and the load, their current prediction values can be respectively achieved by using their historical actual values, through the ultra-short-term prediction algorithm. Then, by a designed allocation principle, the planned output size of wind turbines and diesel generators will be allocated. The control framework is shown in Fig. 2. 


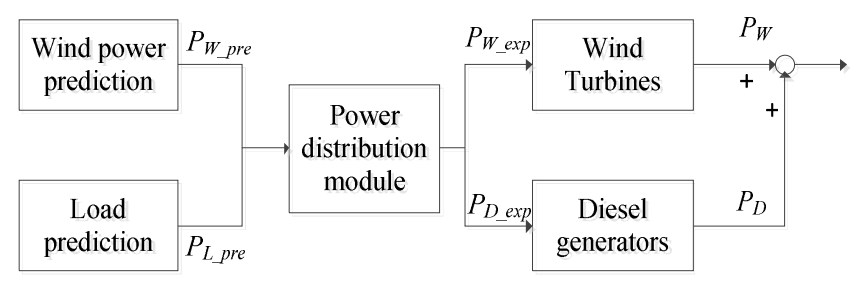

Fig. 2 Feedforword control framework

Here, the core strategy of feedforward part is designed as Eq. 2.

$$
P_{L_{-} \text {pre }}=P_{W_{-} \exp }+P_{D_{-} \exp }=P_{W_{-} \exp }+P_{D}
$$

Where $P_{L_{-} p r e}$ is the prediction value of load, $P_{W_{-} \text {pre }}$ is the prediction value of wind power, $P_{W_{-} e x p}$ is the planned output value of wind turbines, $P_{D_{-} \exp }$ is the planned output value of diesel generators, $P_{W}$ is the actual output value of wind turbines, $P_{D}$ is the actual output value of diesel generators. The error between the planned output value and the actual output value of diesel generators can be ignored, so $P_{D_{-} \exp }$ is equal to $P_{D}$.

Power Distribution Method of Wind Turbine and Diesel Engine. Power distribution is the core in the feedforward part. Its principle is to use wind power as much as possible, and diesel engines work as a supplement. The distribution module is to allocate output power for wind turbines and diesel engines, according to the above principle. Details as follows.

(1) If $P_{W_{-} \text {pre }} \geq P_{L_{-} \text {pre }}$, that means the prediction value of wind power is greater than or equal to the prediction value of the load, $P_{W_{-} \text {exp }}$ is set to $P_{L_{-} \text {pre }}-P_{D_{-} \min }$, and $P_{D_{-} \exp }$ is set to $P_{D_{-} \min }$. But if $P_{W_{-} \exp }>P_{W_{-} a c t_{-} \max }$, that means the planned output of wind power is greater than the actual maximum output of wind power, this part of wind power prediction error will be compensated by the feedback part. If $P_{W_{-} e x p} \leq P_{W_{-} a c t \_m a x}$, that means the planned output of wind power is smaller than or equal to the actual maximum output of wind power, the wind turbines will run at "abandon wind".

(2) If $P_{W_{-} \text {pre }}<P_{L_{-} \text {pre }}$, that means the prediction value of wind power is less than or equal to the prediction value of the load, $P_{W_{-} \text {exp }}$ is set to $P_{W_{-} \text {pre }}$, and $P_{D_{-} \text {exp }}$ is set to $P_{L_{-} \text {pre }}-P_{W_{-} p r e}$. But if $P_{W_{-} \exp }>P_{W_{-} a c t_{-} \max }$, that means the planned output of wind power is greater than the actual maximum output of wind power, this part of wind power prediction error will be compensated by the feedback part. If $P_{W_{-} \text {exp }} \leq P_{W_{-} a c t_{-} \max }$, that means the planned output of wind power is smaller than or equal to the actual maximum output of wind power, the wind turbines will run at "abandon wind".

Given that, diesel engines should not be stopped frequently, the output of diesel engines has to be set a minimum value, which is $P_{D_{-} \min }$ as above.

Design of Feedforward Part. In the feedforward part, there must be the prediction error, including the error between the load prediction value and the load actual value, and the error between the wind power prediction value and the wind power actual value, which will results in active imbalance in the islanded microgrid. Hence, the feedback part must be combined with the feedforward part to eliminate the error. In this part, the classic load frequency control (LFC) method is used, and the prediction error is seen as a disturbance of LFC. Energy storage systems are used to participate in primary frequency modulation and secondary frequency modulation, in order to 
eliminate error timely and gain active balance. As a result, the islanded microgrid frequency is well stabley near the set value. The control framework is shown in Fig. 3.

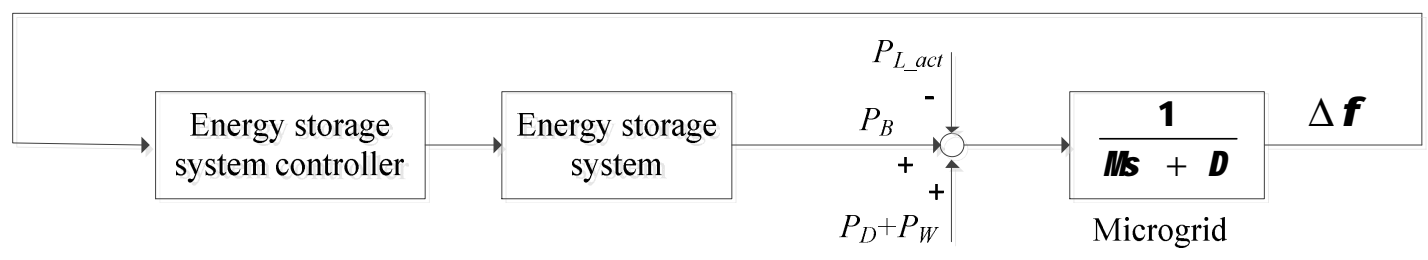

Fig. 3 Feedback control framework

\section{Resources Allocation Method of Feedforward Part and Feedback Part}

The available resources in islanded microgrid is much limited, and unreasonable resources allocation will cause serious waste, which does not accord with the economy of islanded microgrid development. In this paper, each unit in the feedforward and the feedback part must also be optimally configured to make better use of local power generation resources.

Method Description. The unit with large capacity should be arranged in the feedforward part, because the feedforward part is mainly to roughly adjust the load disturbance in advance. At the same time, considering that local renewable energy should be made full use of, new energy units like wind turbines can be arranged in the feedforward part. Then diesel engines with large capacity can be arranged in the feedforward part as a supplement to wind turbines due to the fluctuation of wind. Specifically, the size of the each unit in the feedforward part is related to the local wind power, load fluctuation and the ultra-short-term prediction error.

For the feedback part, this part mainly compensates the prediction error in the feedforward part. It requires that units in the feedback part need to have fast adjustment characteristic. Hence, energy storage systems can be arranged in the feedback part. Specifically, the size of the unit capacity in the feedback part depends on the size of the error after the coarse adjustment in the feedforward part.

Examples. In order to illustrate the method above more vividly, an example is made as follows.

(1) According to the above method, wind turbines and diesel engines are selected in the feedforward part. At the same time, according to the probability distribution of the actual history value of local wind power, the wind turbine capacity is set to $750 \mathrm{KW}$. The capacity of the diesel engines is configured according to the probability distribution of the difference between the historical actual load value and the maximum output power value of wind turbines in the past. Assuming that the capacity of diesel engines must be greater than or equal to the difference between the historical actual load and the maximum output power of wind turbines in $100 \%$ probability, which is $500 \mathrm{KW}$, so the capacity of diesel engine is set to $500 \mathrm{KW}$.

(2) According to the above method, energy storage systems are selected in the feedback part. The capacity of energy storage systems in the feedback part depends on error's size after the coarse adjustment in the feedforward part. For example, according to the historical data, the actual load is $1000 \mathrm{KW}$, the load prediction value is $900 \mathrm{KW}$, the wind power planned value is $750 \mathrm{KW}$, the actual wind power maximum value is $700 \mathrm{KW}$. To ensure that the feedback part can eliminate the prediction error in $100 \%$ probability, the capacity of energy storage systems shall be greater than or equal to $(1000-900)+(750-700)$, so the capacity of energy storage systems is set to $150 \mathrm{KW}$. 


\section{Simulation Results}

Simulation presented in this paper is carried out by Matlab/Simulink.

Simulation Results of Prediction Method. (1) The load is predicted by the moving average method above, and prediction results are shown in Fig. 4.

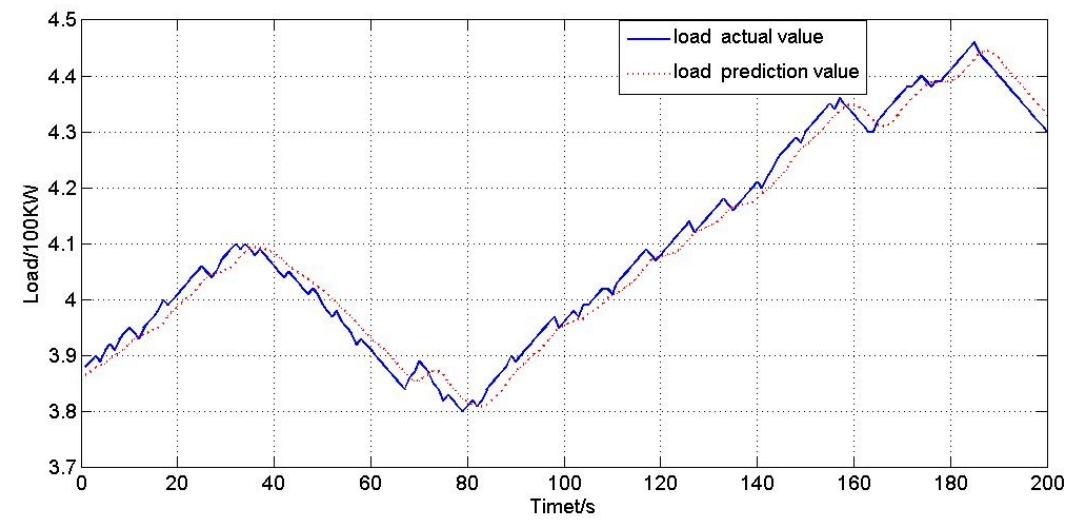

Fig. 4 Load prediction and actual value comparison

From the above results, the prediction error is less than $10 \%$, so the load with small volatility can be well predicted using the moving average method.

(2) The wind power is predicted by the moving average method above, and the prediction results are shown in Fig. 5.

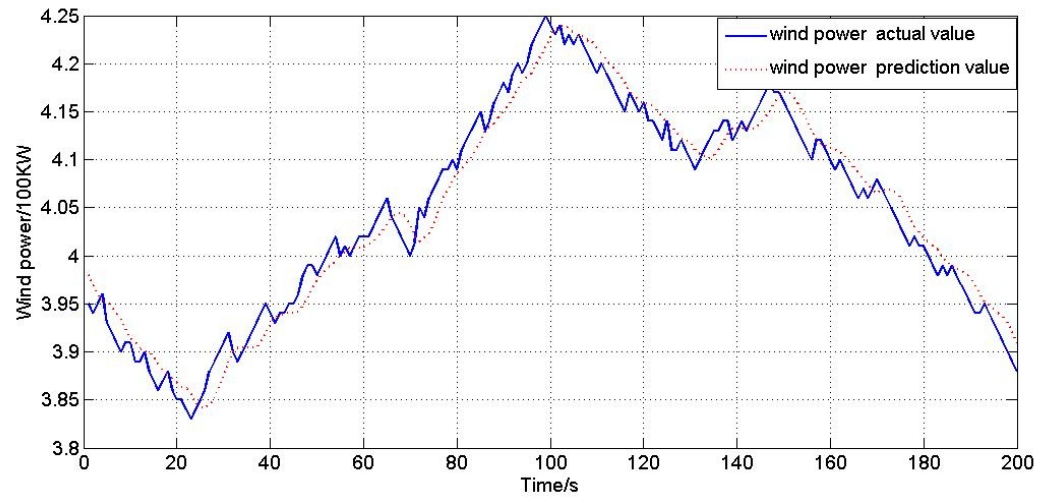

Fig. 5 Wind power prediction and actual value comparison

From the above results, the prediction error is less than $10 \%$, so the wind power with small volatility can also be well predicted using the moving average method.

Simulation Results of Control Methods. In this paper, the configuration of the islanded microgrid generation system is as follows: Wind turbines' rated power is $750 \mathrm{~kW}$, diesel engines' rated power is $350 \mathrm{~kW}$, energy storage systems' rated power is $300 \mathrm{~kW}$, energy storage systems' capacity is $50 \mathrm{~kW} \cdot \mathrm{h}$. These parameters refer to reference [18].

In this paper, two contrast tests are set up. One uses the proposed control method. In the contrast experiment, the wind turbines are running in the maximum power tracking mode all the time and out of control, and only diesel engines and energy storage systems are used to control the islanded microgrid frequency.

The control structure of the contrast method is shown in Fig. 6. 


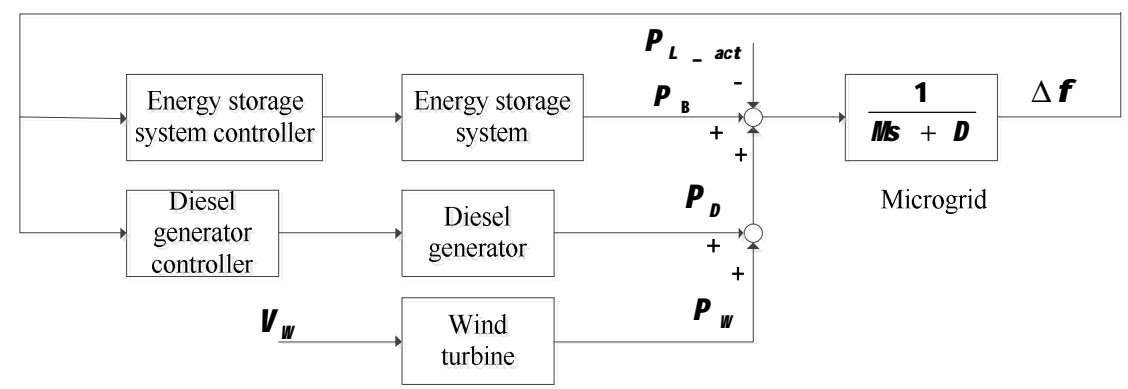

Fig. 6 The control structure of the contrast method

In Matlab, the simulation model of the contrast method is shown in Fig. 7.

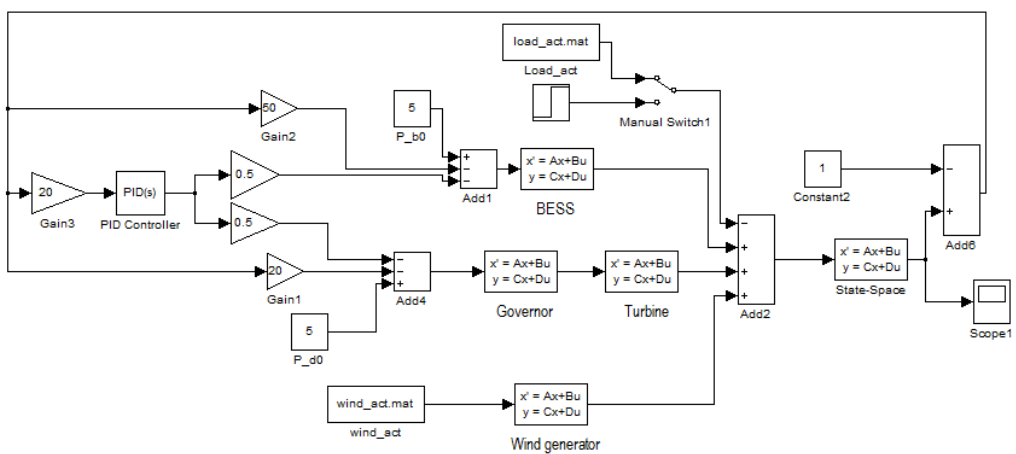

Fig. 7 The simulation model of the contrast method

In Matlab, the simulation model of the proposed method is shown in Fig. 8.

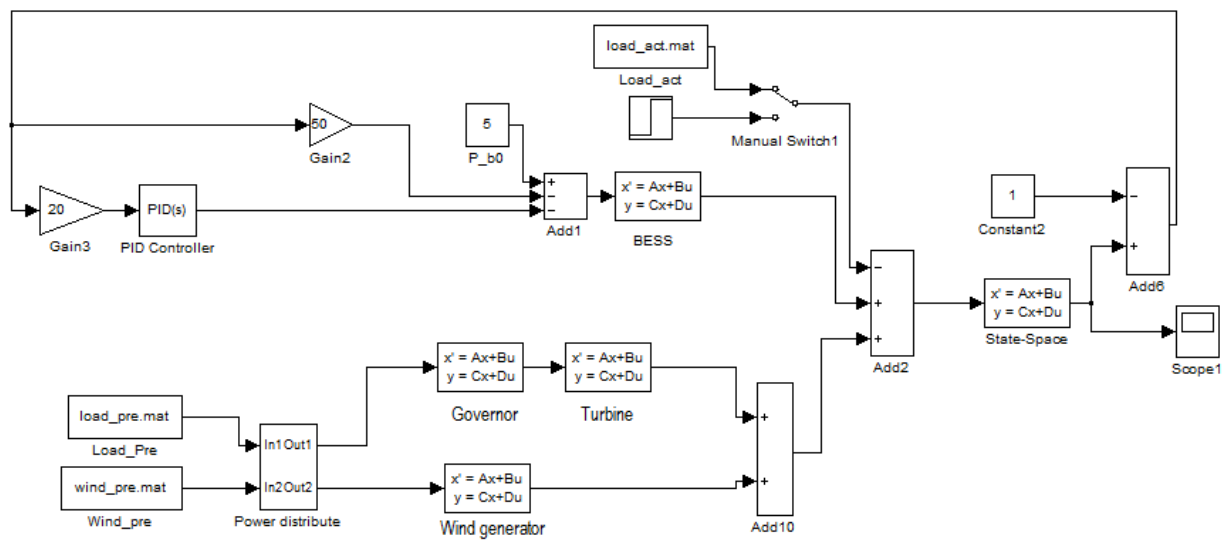

Fig. 8 The simulation model of the proposed method

Through the simulation, the islanded microgrid frequency control results are shown in Fig. 9, and the size of frequency in the figure has been converted into per unitary value. 


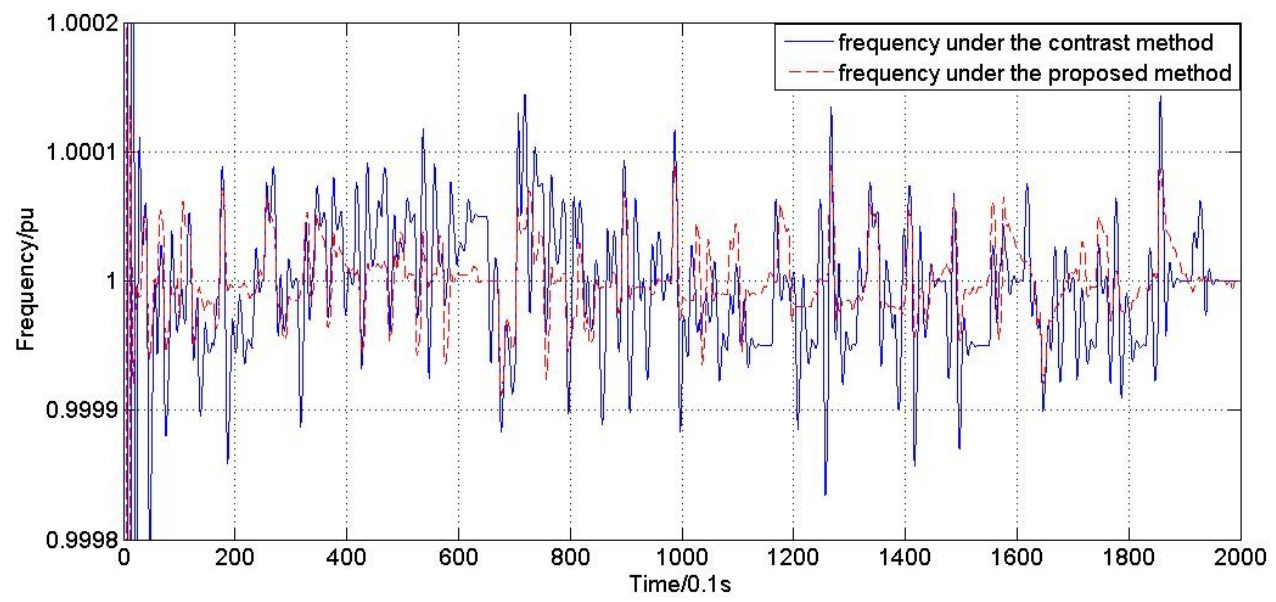

Fig. 9 Islanded microgrid frequency control results comparison

The output results of Energy storage systems are shown in Figure 10 and Figure 11, the size of output has been converted into per unitary value.

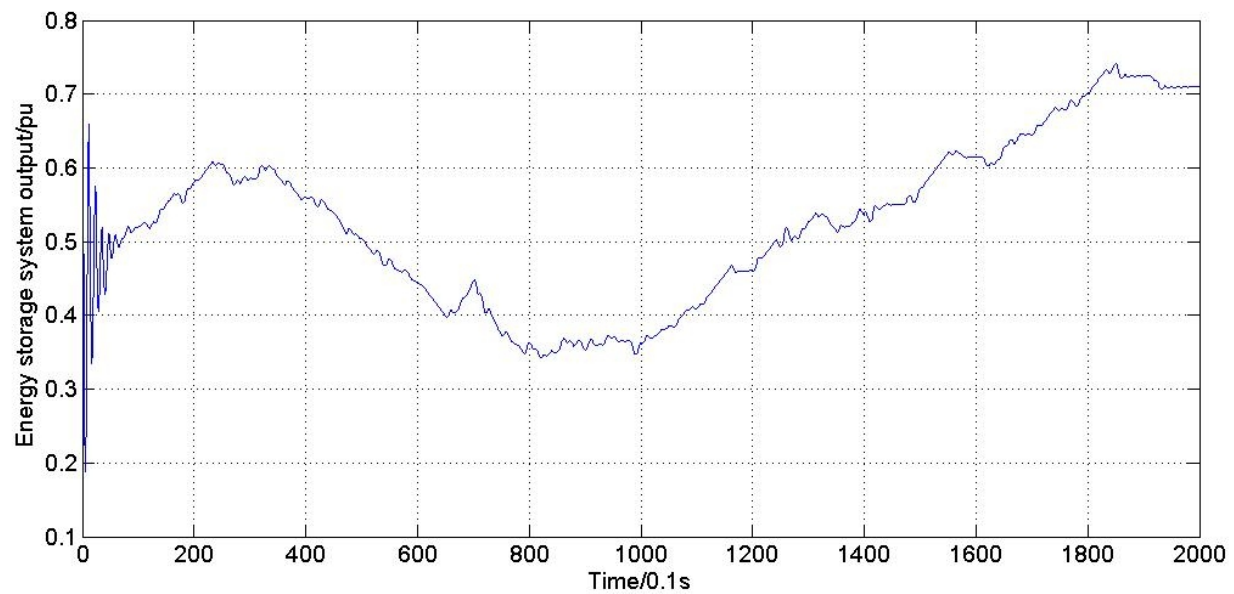

Fig. 10 The output of energy storage system under the contrast control method

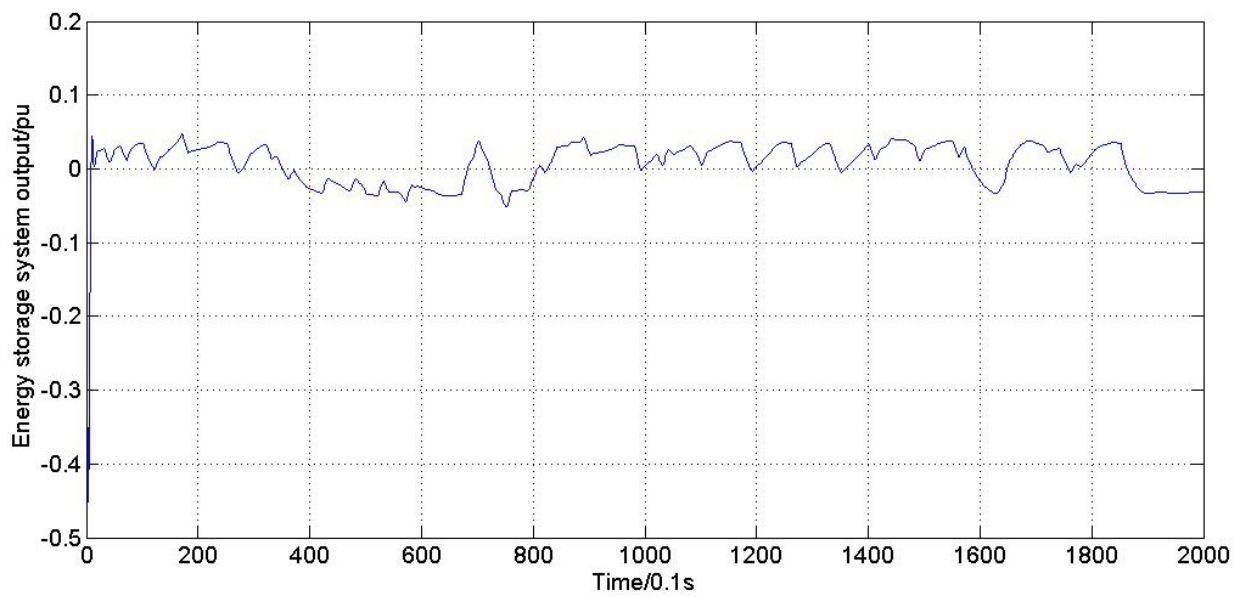

Fig. 11 The output of energy storage system under the proposed control method

It can be seen from Fig. 9 that the system frequency fluctuates around the set value as the load disturbance changes. The fluctuation of the red dotted line is smaller than the blue solid line on the 
whole. It means that the proposed control method has a better effect on suppressing the frequency fluctuation to a certain extent than the traditional control method, and improves the stability of the islanded microgrid. In Fig. 11, the negative value of the vertical axis indicates that the energy storage system is in the charged state. By comparing Fig. 10 and Fig. 11, it can be found that, the output size of the energy storage system under the proposed control method is much smaller than that under the traditional method. It means that through the feedforward and feedback control method, the pressure of energy storage systems in the islanded microgrid is greatly reduced.

\section{Conclusions}

In this paper, the advantages and disadvantages of current islanded microgrid frequency control methods are analyzed. Based on this, the feedforward and feedback frequency control method for islanded microgrid is proposed to improve the frequency control effect of islanded microgrid, and then an allocation method for feedforward and feedback resources is proposed to save construction costs. Simulation results show that the proposed control method can maintain the frequency stability for islanded microgrid better.

\section{Acknowledgements}

This work was financially supported by the National Natural Science Foundation (No. 51577031) and State Grid Corporation of China (Research and Development of Simulation Technology for Medium and Long Term Electricity Trading, DZN17201600244).

\section{References}

[1] Dan LI. An Active Power Control Scheme for Isolated Network Which Included Wind Turbine, PV, Diesel and Battery Based on Stratified Control[D]. University of Electronic Science and Technology, 2013. "In Chinese".

[2] Zhixia WANG, Huimin ZHANG, Wei TIAN. A Summary of Microgrid Research[J]. Electrical Applications, 2010, 06: 76-80. "In Chinese".

[3] Yongwei ZHENG, Minyou CHEN, Chuang LI. Microgrid Control Strategy with Adaptive Adjustment of Droop Coefficient[J]. Power System Automation, 2013, 37(7): 6-11. "In Chinese".

[4] Yunling SUN, Wei HUANG, Guannan WANG. Research on Improvement of Droop Control Strategy Microgrid Connected with Inverter[J]. Shanxi Electric Power, 2013(1): 6-10. "In Chinese".

[5] F Katiraei, M R Iravani. Power Management Strategies for A Microgrid with Multiple Distributed Generation Units[J]. Power Systems, IEEE Transactions on, 2006, 21(4): 1821-1831.

[6] J A Peas Lopes, C L Moreira, A G Madureira. Defining Control Strategies for Microgrid Islanded Operation[J]. Power Systems, IEEE Transactions on, 2006, 21(2): 916-924.

[7] Shanshan SHI, Zongxiang LU. Design of Micropower Power Allocation Strategy in Undifferenced Frequency Modulation[J]. Power System Automation, 2011, 35(19): 23-27. "In Chinese". 
[8] Xiangzhen YANG, Jianhui SU, Ming DING. Frequency Control Strategy of Islanding Microgrid[J]. Power System Technology, 2010,34(1): 164-168. "In Chinese”.

[9] J Y Kim, J H Jeon, S K Kim. Cooperative Control Strategy of Energy Storage System and Microsources for Stabilizing the Microgrid during Islanded Operation[J]. Power Electronics, IEEE Transactions, 2010, 25(12): 3037-3048.

[10]Hajimiragha A H, Dadash Zadeh M R, Moazeni S. Microgrid Frequency Control Considerations Within the Framework of the Optimal Generation Scheduling Problem[J]. IEEE Transactions on Smart Grid, 2014, 6(2):1-1.

[11] Shao LI, Lichen GU, Yu MA. Feedforward - feedback Compound Compensation Control of Variable Speed Hydraulic Power Source[J]. China Mechanical Engineering, 2016, 06: 805-809+832. "In Chinese".

[12] Hongni ZHOU, Li WANG, Jianmin TAO. A Simulation of Vehicle Stability Control System Based on Feedforward - Feedback Compensation Control[J]. Journal of Hubei University of Automotive Technology, 2009, 03: 11-15. "In Chinese".

[13] Chao PAN, Jianmin ZUO, Mulan WANG. High Performance Linear Servo System Based on Feedforward and Feedback Compensation[J]. Journal of System Simulation, 2010, 12: 3025-3029. "In Chinese".

[14] Yingyu LIANG, Jianzheng LIU, Xingtao XU. Three-phase Four-wire APF Control Strategy Based on Feed-forward and Feedback for Power Supply Current and Load Current Detection[J]. Electric Power Automation Equipment, 2015, 01: 94-100. "In Chinese".

[15] Yulin HE, Dongxu SU, Shuai HUANG. Pitch Control and Load Optimization of Variable Speed Pitch Wind Turbine[J]. Power System Protection and Control, 2011, 16: 95-100. "In Chinese".

[16] Xianzheng WANG, Jinqiu MO. Control Theory Foundation[M]. Beijing: Science Press, 2007. "In Chinese".

[17] Dongxiao NIU, Leilei FAN. Overview and Development of Wind Power Forecasting Methods[J]. Modern Electric Power, 2013, 04: 24-28. "In Chinese".

[18] Mendis N, Muttaqi K M, Sayeef S. A Control Approach for Voltage and Frequency Regulation of A Wind-Diesel-Battery based Hybrid Remote Area Power Supply System[J]. IEEE Transactions on Smart Grid, 2010: 3054-3060. 